\title{
„Bei uns hat die 200jährige Jubelfeier viel Staub aufgewirbelt..." Das gescheiterte Gedenkjahr 1877 der Universität Innsbruck
}

\begin{abstract}
The paper examines the failed jubilee of the University of Innsbruck in 1877. Due to a conflict in the senate of the university, the professors were not willing to organize the $200^{\text {th }}$ anniversary celebration of the university's foundation. In this situation, students took over and tried to plan the university's jubilee. However, the students failed in doing so, since they could not reach an agreement whether a catholic Mass should be part of the festivities. For this reason, only some smaller celebrations took place held by student fraternities, which ended in street fights and a scandal. The paper analyses the reasons for the failed jubilee in the context of the public debate on clerical control of the educational system in the Habsburg Monarchy and the discussion on the role of the church in the university at the time of the university's foundation and in the contemporary era. Furthermore, it deals with concepts of cultural memory and performative practices associated with the jubilee.
\end{abstract}

\section{Einleitung}

Das Jahr 2019 stand an der Leopold-Franzens-Universität Innsbruck ganz im Zeichen des 350-Jahr-Jubiläums der Gründung der Universität. Ein ganzes Jahr über standen Festveranstaltungen, Konferenzen, Theateraufführungen, Vorträge sowie Führungen durch die Universitätsgebäude und -einrichtungen auf dem Programm, mit dem unterschiedliche Gesellschaftsgruppen über die Arbeit und die Geschichte der Universität informiert werden sollten. ${ }^{1}$ Eine Historisierung und Aufarbeitung dieses Jubiläumsjahres ist zukünftigen Historikerinnen vorbehalten, in diesem Beitrag soll es stattdessen um das Universitätsjubiläum der Universität Innsbruck im Jahr 1877 gehen, das wenig erfolgreich war und im Streit geendet hatte.

1 Einen Überblick über die Aktivitäten im Jubiläumsjahr bietet die Website der Universität: Universität Innsbruck: 350 Jahre Universität Innsbruck. https://www.uibk.ac.at/350-jahre/ (5.11.2020). 
Noch am 10. April 1877 hatte die Allgemeine Zeitung (Augsburg) angekündigt, dass in Innsbruck ein großes Jubiläumsfest stattfinden werde:

Innsbruck, 7. April (Universitätsjubiläum). Am 25. und 26. des Monats feiert die Innsbrucker Hochschule den Gedächtnißtag ihrer 200jährigen Stiftung durch den Kaiser. Umfassende Vorbereitungen sind dazu bereits getroffen. Namentlich der Fackelzug dürfte sich zu einem Schauspiel gestalten wie Innsbruck es nicht oft gesehen. Die Theilnahme ist allgemein. Am 26. ist Commers. Man hofft, daß die gesammte Universitätsjugend Oesterreichs und auch Deutschlands an diesem Fest ihrer Collegen regen Antheil nehmen werde. ${ }^{2}$

Anders aber als die hoffnungsfrohe Ankündigung in der Allgemeinen Zeitung es erwarten ließ, fiel ein großer Teil des Programms ins Wasser: Die meisten Professoren hatten eine Teilnahme an der geplanten Feier der Studenten wenige Tage zuvor öffentlich abgelehnt, ${ }^{3}$ und der Statthalter hatte den angekündigten Fackelzug der Studenten verboten. ${ }^{4}$ Schließlich lieferten sich einzelne Gruppen der Studentenschaft im Anschluss an getrennte Feiern einen Streit im zentralen Straßenzug der Stadt, der schlussendlich in einer „ordentlichen Holzerei“5 endete, sodass mehrere Studenten „arg zugerichtet ${ }^{\text {"6 }}$ waren, wie danach in den Innsbrucker Nachrichten zu lesen war. Der Rektor der Universität, der Historiker Alfons Huber, schrieb später an einen Kollegen, dass dieses Schauspiel ein peinlicher Skandal für die Universität gewesen sei, der „viel Staub aufgewirbelt“7 habe. Der erste Versuch, ein Jubiläum der Universität zu feiern, war damit gründlich danebengegangen.

Im Folgenden werden die Ursachen und Folgen dieses Scheiterns näher betrachtet und die Faktoren umrissen, die die Planungen und die Feier des Jubiläums beeinflusst haben. Dazu werden der Zeitpunkt und die Umstände des Jubiläums beziehungsweise die historischen Rahmenbedingungen, in die das Jubeljahr fiel, skizziert. In einem zweiten Schritt geht dieser Beitrag auf die Festkultur an der Universität Innsbruck sowie auf die sich entwickelnde Jubiläumspraxis an den Universitäten der Habsburgermonarchie und deren Nachbar-

2 O.V.: Universitätsjubiläum. In: Allgemeine Zeitung Nr. 100 (10.4.1877). S. 1504.

3 Neues Wiener Tagblatt Nr. 110 Abendausgabe (23.4.1877). S. 4; vgl. auch Universitätsarchiv Innsbruck (UAI), Senatssitzungsprotokolle 1862-1885, Karton 4, ad V 1876/77: Rektor Alfons Huber an Ministerium für Cultus und Unterricht, 9.5.1877.

4 Tiroler Landesarchiv (TLA), Statthalterei Präsidium 881/1877: Statthalterei an Theodor Christomannos (Vorsitzender des Studentenausschusses). Entwurf, 24.4.1877.

5 O.V.: Zum Studentenkrawall. In: Innsbrucker Nachrichten Nr. 98 (30.4.1877). S. 1207.

6 O.V., Studentenkrawall (wie Anm. 5).

7 Alfons Huber an Adam Wolf, 22.4.1877. Abgedruckt in: Oberkofler, Gerhard u. Peter Goller (Hrsg.): Alfons Huber. Briefe 1859-1898. Innsbruck/Wien 1995. S. 448. 
ländern ein. Zuletzt wird die Haltung unterschiedlicher universitärer und außeruniversitärer Akteure im Hinblick auf das Jubiläum behandelt und schließlich eine Zusammenschau der Ergebnisse präsentiert.

Mein wesentlicher Ausgangspunkt für den Beitrag ist dabei das Assmann'sche Konzept, ${ }^{8}$ die Feier als Medium der kulturellen Erinnerung zu begreifen. In der historischen Forschung zu Universitäten wurde dieser Ansatz vor allem zur Erinnerungskultur an der Universität Jena genutzt und vertieft. ${ }^{9}$ Daran anknüpfend erscheint mir zentral zu sein, dass über universitäre Jubiläumsfeiern die Vergangenheit der Institution durch die besondere Form der Erinnerung an bestimmte Ereignisse für die Gegenwart nutzbar gemacht werden sollte. Für die Mitglieder der Institution erfüllt das Fest damit eine gemeinschaftsstiftende Funktion zur inneren Festigung und gleichzeitig zur demonstrativen Abgrenzung nach außen. Darüber hinaus gehe ich davon aus, dass sowohl Form als auch Inhalt der Feiern stets Aushandlungsprozessen unterworfen waren, in denen konkurrierende Erinnerungen und damit Gegenwartsentwürfe gegeneinander aufgewogen wurden. Dieser Ansatz wird dabei mit Fragen nach performativen Praktiken, dem doing history, verbunden und untersucht, welche Praxen des Erinnerns für das Jubiläum verwendet wurden, welche Öffentlichkeit(en) damit angesprochen werden sollten und wie die Aushandlungsprozesse dazu verlaufen sind. ${ }^{10}$

8 Assmann, Jan: Der zweidimensionale Mensch. Das Fest als Medium des kollektiven Gedächtnisses. In: Das Fest und das Heilige. Kontrapunkte des Alltags. Hrsg. von dems. u. Theo Sundermeier. Gütersloh 1991 (Studien zum Verstehen fremder Religionen 1). S. 13-30; Assmann, Jan: Das kulturelle Gedächtnis. Schrift, Erinnerung und politische Identität in frühen Hochkulturen. München 1992.

9 Deile, Lars: Feste - eine Definition. In: Das Fest. Beiträge zu einer Theorie und Systematik. Hrsg. von Michael Maurer. Wien/Köln/Weimar 2004. S. 1-17; Halle, Antje: Universitäre Festkultur. In: Traditionen - Brüche - Wandlungen. Die Universität Jena 1850 -1995. Hrsg. von der Senatskommission zur Aufarbeitung der Jenaer Universitätsgeschichte im 20. Jahrhundert. Wien/Köln/ Weimar 2009. S. 254-269; vgl. zur Funktion von Universitätsjubiläen auch: Drüding, Markus: Warum feiern Universitäten Geschichte? Funktionen und Formen deutscher Universitätsjubiläen im späten 19. und 20. Jahrhundert. In: Akademische Festkulturen vom Mittelalter bis zur Gegenwart. Zwischen Inaugurationsfeier und Fachschaftsparty. Hrsg. von Martin Kintzinger, Wolfgang Eric Wagner u. Marian Füssel. Basel 2019 (Veröffentlichungen der Gesellschaft für Universitäts- und Wissenschaftsgeschichte 15). S. 55-76.

10 Vgl. dazu Samida, Stefanie, Sarah Willner u. Georg Koch: Doing History - Geschichte als Praxis. Programmatische Annäherung. In: Doing History. Performative Praktiken in der Geschichtskultur. Hrsg. von Sarah Willner, Georg Koch u. Stefanie Samida. Münster/New York 2016. S. 1-25; Schmidt-Lauber, Brigitta: Die (sich) feiernde Universität. Bedeutungsstiftung durch Jubiläen. In: Doing University. Reflexionen universitärer Alltagspraxis. Hrsg. von Brigitta SchmidtLauber. Wien 2016. S. 55-77. 


\section{Die Rahmenbedingungen}

Die österreichischen Universitäten wurden in Folge der Revolution von 1848 tiefgreifend reformiert. Der Minister für Cultus und Unterricht Leo Thun-Hohenstein verantwortete die Neugestaltung der Habsburger Bildungslandschaft und stärkte die Rolle der Universitäten als Lehr- und Forschungseinrichtungen. Mit der Abschaffung der bisher vorgeschriebenen Lehrbücher, der jährlichen Prüfungen, der Einführung der Lehr- und Lernfreiheit und der Schaffung der Privatdozentur wurden zahlreiche liberale Reformschritte gesetzt. ${ }^{11}$ Vorbild für die Reformen waren die deutschen Universitäten, allen voran die Universitäten Preußens, die seit den Napoleonischen Kriegen selbst einen Reformprozess durchgemacht hatten und als „blühende Hochschulen Deutschlands“12 nun eine Orientierung für die Reform in Österreich darstellten. Wenngleich es dabei durchaus zu einer differenzierten Anpassung gekommen war, hatten die prononcierte Betonung des deutschen Vorbilds sowie die zahlreichen Berufungen von Professoren und Dozenten aus den benachbarten Staaten des Deutschen Bundes im Zuge der Umsetzung der Reformen in den 1850er Jahren zu einer intensiven Debatte um den Verlust der eigenen, österreichischen Universitätstradition geführt. Hinzu kam, dass besonders konservative und klerikale Kreise in der „preußischen Fortschritterei“"13 und den liberalen Reformen eine Abkehr von den katholischen Wurzeln der österreichischen Universitäten erblickten. ${ }^{14}$ Dies galt für die Habsburgermonarchie im Allgemeinen, aber auch für die Innsbrucker Universität im Speziellen.

In Innsbruck war die Situation überdies noch etwas verworrener, denn in Tirol, dem „Kirchenstaat Habsburgs“"15, wurde parallel und in Reaktion auf die liberalen Reformen die Errichtung einer katholischen Universität diskutiert und

11 Vgl. zum Reformprozess zuletzt und dort mit ausführlichen Literaturverweisen: Aichner, Christof u. Brigitte Mazohl (Hrsg.): Die Thun-Hohenstein'schen Universitätsreformen 1849-1860. Konzeption - Umsetzung - Nachwirkungen. Wien/Köln/Weimar 2017.

12 Aus einer Rede des ersten Unterrichtsministers Karl von Sommaruga im März 1848, abgedruckt bei: Heintl, Carl: Mittheilungen aus den Universitäts-Acten (vom 12. März 1848 bis 22. Juli 1848). Wien 1848. S. $10-11$.

13 Diözesanarchiv Wien, Bischofsakten Rauscher, 1860: Karl Kopetzky an Kardinal Joseph Othmar Rauscher. 30.12.1860.

14 Vgl. dazu bei Aichner, Christof: Die Universität Innsbruck in der Ära der Thun-Hohenstein'schen Reformen 1848-1860. Aufbruch in eine neue Zeit. Wien/Köln/Weimar 2018 (Veröffentlichungen der Kommission für Neuere Geschichte Österreichs, Bd. 117). S. 79-87, S. 392- 400. 15 Klieber, Rupert: Jüdische, christliche, muslimische Lebenswelten der Donaumonarchie 1848 1918. Wien 2010. S. 131. 
schließlich mit der Wiedereröffnung der Theologischen Fakultät und deren Übertragung an die Jesuiten im Jahr 1857 ein entschiedener Gegenpunkt zu den liberalen Reformen des Jahres 1848 gesetzt. Die Frage der Rolle und der Vorrechte der Kirche innerhalb der Universität wurde damit neu aufgeworfen und es kam zu einer intensiven Debatte zwischen jenen, die die absolute Freiheit der Wissenschaft forderten und jenen, die die Rolle der Religion und der Kirche für die Wissenschaft und die Universität betonten. Diese Diskussion fand dabei sowohl innerhalb der Universität statt und führte dort zu zahlreichen Konflikten, fand ihren Niederschlag aber auch im Tiroler Landtag und in regionalen und Monarchie-weiten Zeitungen. ${ }^{16}$

Daneben zeigte sich in diesen Debatten auch ein Spannungsfeld zwischen den Vorstellungen von der Universität als einer regionalen Bildungs- und Forschungsstätte mit traditionellen Rechten einerseits und einer zentral von Wien gelenkten Staatsanstalt mit gesamtstaatlichem Auftrag andererseits. Diese unterschiedlichen Vorstellungen von der Funktion der Universität sind in den 1860er Jahren immer wieder in einzelnen Episoden greifbar und flammten im Jubiläumsjahr öffentlichkeitswirksam auf.

Neben diesen Konflikten bildet die Festkultur an der Universität Innsbruck und die sich etablierende Jubiläumskultur im späten 19. Jahrhundert, der „era of jubilees“17, an den deutschen und österreichischen Universitäten eine wichtige Rolle für das Verständnis der Dynamiken im Jubiläumsjahr. Für eine Jubiläumsfeier gab es in Innsbruck selbst kein Vorbild - allerdings für das Scheitern eines Jubiläums, wie die Innsbrucker Nachrichten am 12. März 1877 mit Blick auf die sich abzeichnenden Schwierigkeiten bei der Planung für das anstehende Jubiläum süffisant berichteten: Denn im Jahr 1773 war der „Antrag einer Säkularfeier“ wegen des gerade erfolgten Verbots des Jesuitenordens, der bis dahin die Universität maßgeblich geprägt hatte, fallen gelassen worden. ${ }^{18}$

16 Vgl. dazu Aichner, Christof: Die Verbindung von Lehre und Forschung - auf dem Weg zur modernen Universität im 19. Jahrhundert. In: Geschichte der Universität Innsbruck 1669-2019. Bd. I: Phasen der Universitätsgeschichte. Teilbd. 1: Von der Gründung bis zum Ende des Ersten Weltkriegs. Hrsg. von Margret Friedrich u. Dirk Rupnow. Innsbruck 2019. S. 295-470, hier S. 372378; Oberkofler, Gerhard: Die Petition der drei weltlichen Fakultäten um Aufhebung der Jesuitenfakultät von Jahr 1873. Ein Beitrag zur Geschichte des Kampfes zwischen kirchlichem und freiem Denken an der Universität Innsbruck. In: Tiroler Heimat 36 (1973). S. $77-91$.

17 Drüding, Universitäten (wie Anm. 9), S. 60; allgemein dazu auch: Müller, Winfried: Inszenierte Erinnerung an welche Traditionen? Universitätsjubiläen im 19. Jahrhundert. In: Die Berliner Universität im Kontext der deutschen Universitätslandschaft nach 1800, um 1860 und um 1910. Hrsg. von Rüdiger vom Bruch. Unter Mitarbeit von Elisabeth Müller-Luckner. München 2010 (Schriften des Historischen Kollegs, Kolloquien 76). S. 73-92.

18 O.V.: Von der Universität vor 100 Jahren. In: Innsbrucker Nachrichten Nr. 58 (12.3.1877). S. 695. 
Indes gab es wie an anderen Universitäten jährlich wiederkehrende Feierlichkeiten, wobei besonders die Feiern zur Erinnerung an die „Restauration“ ${ }^{\text {“19 }} \mathrm{der}$ Universität im Jahr 1826 genannt werden können. In den Jahren nach 1826 wurde dieses Ereignis alljährlich in einem Festakt in zeitlicher Nähe zum 30. April, dem Tag der Restauration, erinnert. Der Festakt bestand aus einem Gottesdienst mit anschließender Rede des Rektors in der Aula. ${ }^{20}$ In der Reformära nach 1848 verlor diese Praxis allerdings an Bedeutung, sodass der Senat das Fest zu Beginn der 1870er Jahre auch formal abschaffte. Die Senatsmehrheit hatte dies mit dem Bedeutungsverlust des Festes begründet, zumal die Erinnerung an die Restauration durch die jüngsten Reformen überholt schien, sowie mit der Tatsache, dass durch das Fest ein Vorlesungstag verlorengehe. Die Minderheit im Senat argumentierte hingegen damit, dass man mit der Feier die Möglichkeit habe „nach außen Zeugnis zu geben“. ${ }^{21}$ Jenen, die zur Optimierung des Studienbetriebs einen Ferialtag abschaffen wollten, stand somit eine kleinere Gruppe entgegen, die diese Chance zur öffentlichen Repräsentation der Universität nicht aufgeben wollte. Ähnlich wie letztere Gruppe hatte bereits in den 1850er Jahren der konservative Rechtswissenschaftler Ernst von Moy de Sons gefordert, die noch immer wirksamen josephinischen Rationalisierungsmaßnahmen (beispielsweise die Abschaffung der Talare) rückgängig zu machen und akademische Feiern wieder mit größerem Aufwand zu feiern, um in diesen öffentlichen Akten die universitäre Gemeinschaft zu stärken und die Sichtbarkeit der Universität in der Gesellschaft zu erhöhen. ${ }^{22}$

Daneben hatte es 1857 sowie 1869 bei der Erweiterung der Universität durch die Theologische respektive die Medizinische Fakultät große Feiern gegeben, die jeweils auch Positionsbestimmungen gewesen waren, die gegensätzlicher nicht hätten sein können: Während 1857 die Übergabe der Theologischen Fakultät an die Jesuiten als ein Anknüpfen an den historischen Ursprung der Universität ge-

19 Im Jahr 1826 wurde die Universität, nachdem sie zuvor für einen längeren Zeitraum zu einem Lyzeum herabgestuft worden war, wieder in den Rang einer Universität gehoben und erhielt das Promotionsrecht zurück. Im zeitgenössischen Sprachgebrauch wurde stets von der ,Restauration' gesprochen.

20 Vgl. dazu Aichner, Verbindung (wie Anm. 16), S. 298-299.

21 UAI, Senatssitzungsprotokolle 1862-1885, Karton 4, II 1873/74: Senatssitzung vom 27.1.1874. 22 UAI, Juridische Sitzungsprotokolle 1848-1871: Sitzungsprotokolle der juridischen Fakultät, Nr. 311, 20.1.1860 u. Nr. 440, 19.6.1860; vgl. allgemein zur Funktion universitärer Feierlichkeiten, in denen sich die Aussagen von Moy de Sons widerspiegeln bzw. den Wunsch nach einer Rückkehr zu traditionellen Formen akademischer Feiern unterstreichen: Füssel, Marian: Akademische Solennitäten. Universitäre Festkulturen im Vergleich. In: Festkulturen im Vergleich. Inszenierungen des Religiösen und Politischen. Hrsg. von Michael Maurer. Köln/Weimar/Wien 2010. S. 43-60, hier vor allem S. 44. 
feiert und die Universität als Bollwerk des katholischen Glaubens apostrophiert wurde, stand die Feier zur Eröffnung der Medizinischen Fakultät 1869 ganz im Zeichen des Glaubens an einen ungebremsten Fortschritt durch Wissenschaft, mit der Universität als Garant für Freiheit und Aufklärung. ${ }^{23}$

Schließlich fallen in die 1860er Jahre auch mehrere Jubiläen von Universitäten im In- und Ausland, zu denen Vertreter der Innsbrucker Universität eingeladen waren (Berlin, Basel, Wien, München, Leiden). ${ }^{24}$ Der Senat diskutierte dabei jeweils darüber, wie man auf diese Einladungen reagieren und ob sich die Universität an den Festakten beteiligen solle. Besonders intensiv erörterte der Senat im Frühjahr 1872 die Einladung zum anstehenden Münchner Universitätsjubiläum. Während ein Großteil der Mitglieder des Senats sich enthusiastisch für eine aktive Beteiligung und das Überreichen von Festschriften an die „Nachbaruniversität“ ${ }^{25}$ einsetzte, sprach sich die Theologische Fakultät geschlossen gegen eine Teilnahme aus. Sie begründete dies damit, dass die Feier in München, wie in der Einladung angekündigt worden war, ein Fest der noch jungen deutschen Nation werden sollte („Das Fest wird Zeugnis geben wie die Nation zu den Universitäten und die Universitäten zu der Nation stehen“26). Die Theologische Fakultät befürchtete, dass ,der Geist in welchem die Feier begangen werden soll, ein der katholischen Kirche feindseliger [sei] oder wenigstens in einer oder der anderen Rücksicht antikatholische Tendenzen dabei das leitende Princip bilden“ würden. ${ }^{27}$ Während die weltlichen Fakultäten unter der Führung des Rektors Kamill Heller (Medizinische Fakultät) die Gelegenheit nutzen wollten, um zu demonstrieren, dass „,die scheidenden staatlichen Grenzen [...] ohne Einfluß auf die Beziehungen der beiden Hochschulen“ seien und dass „die deutsche Wissenschaft [...] überall Eine [!] trotz trennender Berge und Ströme und Grenzen“28 sei, wollten die Theologen keine derartige öffentliche Positionierung der Innsbrucker Universitäten unterstützen. Da die Senatoren der Theologischen Fakultät aber in der Minderheit waren, konnten sie sich mit ihren Bedenken nicht durchsetzen und die Universität sandte eine Deputation, ausgestattet mit einer Glückwunschadresse und zwei Festschriften, nach München.

23 Vgl. dazu Aichner, Verbindung (wie Anm. 16), S. 368-372.

24 UAI. Karton Festlichkeiten, Jubiläen 1.

25 UAI, Akten des Rektorats, ad 428/R 1871/72: Senat der LFU an die drei weltlichen Fakultäten, 11.4.1872.

26 UAI, Akten des Rektorats, 428/R 1871/72: Einladung zur Münchener Säcularfeier.

27 UAI, Senatssitzungsprotokolle 1862-1885, Karton 4, III 1871/72: Stellungnahme der Theologischen Fakultät der LFU in der Senatssitzung vom 8.3.1872.

28 UAI, Akten des Rektorats, ad 428/R 1871/72: Grußadresse an die Universität München, 15.7.1872 (Entwurf). 
Fasst man nun die Rahmenbedingungen für das Jubiläum der Innsbrucker Universität im Jahr 1877 zusammen, so kann man festhalten, dass seit den 1850er Jahren innerhalb der Universität mehrere Konflikte schwelten, die in erster Linie aus unterschiedlichen Vorstellungen von der Stellung der Universität zur Kirche und von der Rolle von Religion und Kirche in den Wissenschaften resultierten. Akademische Feiern waren in diesem Sinn wichtige Veranstaltungen, bei denen einzelne akademische Gruppen oder Professoren die Universität im jeweiligen Sinn positionieren konnten. Bei mehreren solchen Ereignissen zeichneten sich dabei schon jene Entwicklungen und Konflikte ab, die im Jubiläumsjahr besondere Wirksamkeit entfalten sollten.

\section{Das Jubiläumsjahr 1877}

Da das Bedürfnis nach öffentlicher Positionierung in dieser Umbruchphase groß war, und obschon - wie die Debatten anlässlich der Einladungen zu Jubiläen anderer Universitäten gezeigt hatten - dem Senat die Möglichkeiten eines Jubiläums für die Selbstdarstellung durchaus bewusst waren, erstaunt es umso mehr, dass die Universität Innsbruck vom eigenen Jubiläum letztlich überrascht wurde. Im November 1876 wies die Wiener Neue Freie Presse nämlich darauf hin, dass ein Jubiläum der Universität Innsbruck anstehe. Weiter vermeldete die Zeitung, dass bereits eifrig geplant werde und das Jubiläum „zu einem wahren Universitätsfeste“29 gestaltet werden solle. Wie der Rektor Alfons Huber dem Unterrichtsministerium aber retrospektiv mitteilte, hatte es zu diesem Zeitpunkt keine Planungen oder Vorbereitungen gegeben. ${ }^{30}$ Allerdings, so Rektor Huber in derselben Erklärung, befand sich die Universität nun in einem gewissen Zugzwang, sich zu äußern und tatsächlich eine Feier zu veranstalten. Da der Senat zu diesem Zeitpunkt jedoch aufgrund von Streitigkeiten weitgehend handlungsunfähig war, beschloss der Rektor die Sache zunächst nicht weiter zu verfolgen, was sich später rächen sollte, als die Studenten, die ebenfalls die Meldungen in der Zeitung verfolgt hatten, dieses Versäumnis des Senats zu kompensieren versuchten und selbst an die Organisation einer Jubiläumsfeier schritten.

Bei der Analyse dieser Planungen lassen sich unterschiedliche Vorstellungen davon identifizieren, welches Jahr oder Datum überhaupt als Referenzpunkt für ein Jubiläum zu gelten hatte. Im angesprochenen Zeitungsartikel aus dem No-

29 O.V.: Eine verwaiste Universität. In: Neue Freie Presse Nr. 4394 Abendblatt (17.11.1876). S. 1. 30 UAI, Senatssitzungsprotokolle 1862-1885, Karton 4, ad V 1876/77: Rektor Alfons Huber an Ministerium für Cultus und Unterricht, 9.5.1877. 
vember 1876 bezog man sich nämlich auf das Jahr 1826, also auf die oben erwähnte Restauration der Universität. ${ }^{31}$ Dies hätte ein halbes Zentenarium bedeutet. Da im November eine Planung für eine Feier im selben Jahr wohl nicht mehr machbar war, kam es den Studenten durchaus zupass, dass in das Jahr 1877 die 200. Wiederkehr der kaiserlichen (26. April 1677) und päpstlichen (28. Juli 1677) Stiftungsurkunden fielen. Man griff somit den Vorschlag zwar auf, deutete ihn aber um. Dies bot den Vorteil, dass man einerseits noch ein wenig Vorbereitungszeit hatte und andererseits eine längere Geschichte - 200 Jahre versus 50 Jahre - feiern konnte. Allerdings war auch dieses Datum nicht unumstritten, wie ein Artikel im liberalen Neuen Wiener Tagblatt beweist. Dort wird vielmehr auf das Jahr 1673 verwiesen, in dem die Universität das Promotionsrecht erhalten hatte, sodass die Zeitung dem Jubiläum 1877 jegliche Berechtigung absprach. ${ }^{32}$ Dass mit den unterschiedlichen Referenzpunkten auch unterschiedliche Vorstellungen vom Status der Universität verbunden waren, wird später noch gezeigt werden.

Heute, und damit sei ein letzter Hinweis zu dieser Thematik ergänzt, gilt das Jahr 1669 als Ursprungsjahr der Innsbrucker Universität. Am 15. Oktober 1669 genehmigte Kaiser Leopold nämlich eine Sondersteuer auf in Hall in Tirol abgebautes und in Tirol verkauftes Salz zur Finanzierung der zu gründenden Landesuniversität. ${ }^{33}$

\section{Die Akteure}

Der Senat war aufgrund eines Konflikts wegen der Auflösung einer studentischen Korporation so zerstritten, dass Rektor Alfons Huber die Feier eines Jubiläums ausschloss und die Mehrheit des Senats eine Feier bei „den gegenwärtigen Zuständen für inopportun“334 hielt. In dieser Situation ergriffen die Studenten Anfang

31 Vgl. Aichner, Verbindung (wie Anm. 16), S. 295-299.

32 O.V.: Zum Universitätsjubiläum in Innsbruck. In: Neues Wiener Tagblatt Nr. 67 (10.3.1877). S. 4. 33 Vgl. dazu Goller, Peter: Innsbrucker Universitätsjubiläen. Inszenierungen 1877 - 1927 - 1952 1969. In: Wissenschafts- und Universitätsforschung am Archiv. Beiträge anlässlich des Österreichischen Universitätskolloquiums am 14. und 15. April 2015 zu den Fragen: Historische Wissenschaftsforschung, Universitäten im gesellschaftlichen Kontext, Internalistische Wissenschaftsgeschichte, Disziplinen- und Institutionengeschichte. Hrsg. von Alois Kernbauer. Graz 2016 (Publikationen aus dem Archiv der Universität Graz 45). S. 69-90.

34 UAI, Senatssitzungsprotokolle 1862-1885, Karton 4, ad V 1876/77: Rektor Alfons Huber an Ministerium für Cultus und Unterricht, 9.5.1877; dieser Bericht stellt im Übrigen zusammen mit den Senatsprotokollen die wichtigste Quelle für die Vorgänge dar, da es keine Aufzeichnungen von den Studentenversammlungen gibt. 
März 1877 die Initiative und beschlossen in einer Versammlung, selbst eine Feier zu organisieren. Sie begründeten dies damit, wegen des Versäumnisses der Professoren gewissermaßen ,gezwungen [zu sein], diesen denkwürdigen Tag in nur studentischer Weise zu feiern, - getragen von der festen Überzeugung, dass es unter solchen Umständen wenigstens unsere Aufgabe sei, einen solchen Tag nicht unberücksichtigt vorbeigehen zu lassen. “35

Da bei der ersten Beratung die Studenten der Theologischen Fakultät und die Vertreter katholischer Studentenverbindungen in der Mehrheit waren, hatte die Versammlung beschlossen, auch einen Gottesdienst in das Festprogramm aufzunehmen und

den Studierenden der theologischen Facultät im Festcomité sechs und nicht wie den anderen Facultäten je vier Vertreter einzuräumen, da die andern Facultäten dadurch mehr als entschädigt wurden, daß die wissenschaftlichen Vereine, Corps, Landmannschaften usw. auch je einen Vertreter wählen durften. ${ }^{36}$

Vor allem die Studenten der Juridischen und der Medizinischen Fakultät fühlten sich durch diesen Beschluss brüskiert. Sie fürchteten, dass die Feier „als eine clericale Demonstration“"37 angesehen werden würde und die Universität so in ein falsches Licht gerückt werde. Daher zogen sich diese Studenten unter der Meinungsführerschaft der liberal und großdeutsch eingestellten Corps zunächst von der weiteren Organisation der Feier zurück. ${ }^{38}$ Einige Professoren versuchten, die übrigen Studenten davon abzubringen, eine Feier zu veranstalten. Diese demonstrierten allerdings gegen dieses „Eingreifen eines Theiles der Professoren“39 und setzten die Planungen fort.

Anders als die Professoren konnten sich die Studenten in der Folge auf einen Kompromiss einigen. Dieser bestand darin, dass der Festgottesdienst nunmehr ausschließlich von der Theologischen Fakultät veranstaltet wurde. Überdies sah das neue Festprogramm einen Fackelumzug mit Illumination der Universität am

35 TLA, Statthalterei Präsidium 881/1877: Theodor Christomannos (Vorsitzender des Studentenausschusses) an Statthalter, 24.4.1877.

36 UAI, Senatssitzungsprotokolle 1862-1885, Karton 4, ad V 1876/77: Rektor Alfons Huber an Ministerium für Cultus und Unterricht, 9.5.1877.

37 UAI, Senatssitzungsprotokolle 1862-1885, Karton 4, ad V 1876/77: Rektor Alfons Huber an Ministerium für Cultus und Unterricht, 9.5.1877; vgl. dazu auch TLA, Statthalterei, Präsidium, 51/ 1877: Statthalter Eduard Taafe an Ministerium für Cultus und Unterricht, o. D. [Mai 1877], Entwurf. 38 Vgl. dazu auch die Schilderung in: o.V.: Zur Universitäts-Affaire. In: Innsbrucker Tagblatt Nr. 56 (9.3.1877). S. 3.

39 UAI, Senatssitzungsprotokolle 1862-1885, Karton 4, ad V 1876/77: Rektor Alfons Huber an Ministerium für Cultus und Unterricht, 9.5.1877. 
Vorabend des Festes und am Festtag selbst einen Empfang mit Reden in der Aula der Universität vor. ${ }^{40}$ Auch wenn die Studenten nach außen damit eine gewisse Einigkeit zeigten, konnten der Kompromiss und die nachfolgenden Ereignisse nicht darüber hinwegtäuschen, dass es sich letztlich um zwei getrennte Feiern an zwei Tagen handelte, deren Bruchlinie entlang der unterschiedlichen Vorstellungen von der Stellung der Universität zur katholischen Kirche verlief.

Während die Theologen und die katholischen Verbindungen weiterhin den Festgottesdienst in den Mittelpunkt stellten und damit die Tradition bisheriger akademischer Feiern und den katholischen Ursprung der Universität betonten, brach der Programmteil, für den die Corps verantwortlich zeichneten, bewusst mit dieser Tradition und bemühte alternative Traditionsstränge. Besonders der geplante Fackelumzug hatte zwar grundsätzlich eine lange studentische Tradition, besaß in der jüngeren Vergangenheit aber vor allem in der burschenschaftlichen Festkultur eine wichtige Rolle. ${ }^{41}$ So standen sich die jeweils geplanten festlichen Höhepunkte der getrennten Feiern und damit die Form und das Publikum, das damit adressiert wurde, entgegen: Im Gottesdienst in erster Linie die akademische Gemeinschaft - im Fackelumzug die städtische Öffentlichkeit. Besonders in der Symbolik unterschieden sich die beiden Feiern aber. Auf der einen Seite die althergebrachte katholische Messe, auf der Gegenseite der Fackelumzug durch die Stadt, mit seinen burschenschaftlichen Bezügen und seiner Lichtsymbolik. Dieses „Licht der Aufklärung“ sollte, wie es das Wiener Tagblatt forderte „das Köhlerlicht der Jesuitenfakultät ${ }^{\text {42 }}$ ersetzen.

Wesentlich für das Verständnis der Vorgänge in der Planungsphase ist die Rolle der Presse, und damit in weiterem Sinne der Öffentlichkeit, zu diesen Konflikten. Die Presse hatte die Streitigkeiten prompt aufgegriffen und dafür gesorgt, dass die Versuche des Rektors, den universitätsinternen Streit klein zu halten, misslangen. Mit Kommentaren von Seiten liberaler Blätter ${ }^{43}$ auf der einen und konservativ-klerikalen Zeitungen ${ }^{44}$ auf der anderen Seite heizte die Presse

40 Vgl. o.V.: Innsbrucker Universitätsfeier. In: Neue Freue Presse Nr. 94 Abendblatt (7.4.1877). S. 13.

41 Vgl. auch die Überlegungen bei Dücker, Burckhard: Fackelzüge als akademische Rituale. In: Zeitschrift für Literaturwissenschaft und Linguistik 36 (2006). S. 105-128.

42 O.V., Universitätsjubiläum (wie Anm. 32).

43 Vgl. mehrere Kommentare im Innsbrucker Tagblatt Nr. 56 (9.3.1877). S. 3; o.V.: Eine „katholische“ Universität. In: Neue Freie Presse Nr. 4503 Abendblatt (10.3.1877). S. 2-3; o.V.: Zum Universitätsjubiläum in Innsbruck. In: Neues Wiener Tagblatt Nr. 67 (10.3.1867). S. 4; o.V.: Die Schwarzen an der Universität Innsbruck. In: Neues Wiener Tagblatt Nr. 110 Abendblatt (23.4. 1877). S. 4.

44 O.V.: Innsbruck. In: Grazer Volksblatt Nr. 60 (15.3.1877). S. 2; o.V.: Akademisches. In: Linzer Volksblatt Nr. 62 (17.3.1877). S. 3. 
den Konflikt weiter an, indem sie entweder das Verhalten der einen Gruppe von Studenten oder der anderen als falsch und nicht als der Tradition und der Bestimmung der Universität angemessen bezeichnete. Gleichzeitig benutzten beide studentischen Seiten die Presse als Forum, um die jeweils eigene Position in eingesandten Kommentaren öffentlich zu ventilieren. Überdies gibt es mehrere Zeitungsberichte, die Interna der Studentenversammlungen ausbreiteten und wohl von Studenten selbst an die Zeitungen weitergegeben wurden. Offenbar bestand auf Seiten der jeweiligen studentischen Gruppen der Wunsch, die Debatte in die Öffentlichkeit zu tragen und so die Deutungshoheit zu erlangen. ${ }^{45}$ Somit erfolgte die Aushandlung über die richtige Form der Feier und damit der Repräsentation der Universität nun auch in der Öffentlichkeit.

Das liberale Neue Wiener Tagblatt griff die geplante Feier der Studenten heftig an und stellte die gesamte Feierlichkeit mit dem Argument in Frage, dass man mit dem Rückgriff auf die päpstliche Stiftung im Jahr 1677 an ein Ereignis erinnere, das sich nicht für ein Gedenken eigne. Damit gedenke man einer „Epoche der Menschen-Unwürde, des Fanatismus und der geistigen Knechtschaft" ${ }^{* 6}$ durch die Jesuiten, die damals die Universität dominierten. Das Tagblatt stellte dabei auch einen Bezug zur Gegenwart her, in welcher Papst Pius IX. mit dem Syllabus errorum ebenfalls geistige und gesellschaftliche Entwicklungen der Zeit verurteilt hatte: „Es ist ein trauriges Zeichen der Zeit, daß man heute die Konvalidirung des ursprünglichen Berufes unserer Hochschule durch den Papst feierlich begehen kann, daß man die Zumuthung an Professoren und Studenten stellt, an so einem Feste theilzunehmen. “47 Stattdessen vertrat der Artikel die Ansicht, dass das eigentliche Jubiläum vier Jahre zuvor, 1873 (wohl in Erinnerung an die Verleihung des Promotionsrechtes 1673) hätte gefeiert werden müssen, man damals aber gut daran getan habe, dieser dunklen Epoche der jesuitischen Dominanz an der Universität nicht zu gedenken. Auch ein Kommentar im Innsbrucker Tagblatt, und damit in einem Blatt mit vorwiegend regionaler Reichweite, sah in der beklagten Vereinnahmung der Universität durch klerikale Kreise, einen „groben Irrthum“48 und eine „Maßlosigkeit klerikalen Fanatismus“. Denn dadurch leugne man, dass

45 Siehe dazu beispielhaft die drei Kommentare im Anschluss an die Sitzung der Studenten, in der der Konflikt ausgebrochen ist: Innsbrucker Tagblatt Nr. 56 (9.3.1877). S. 3; unterzeichnet sind die Kommentare mit: „A. Christ, stud. phil.“, „Mehrere Erz-Philister“ und „Einige Freunde konfessionsloser Wissenschaft".

46 O.V., Eine „katholische“ Universität (wie Anm. 43), S. 2.

47 O.V., Eine „katholische“ Universität (wie Anm. 43), S. 2.

48 O.V.: Innsbruck. In: Innsbrucker Tagblatt Nr. 56 (9.3.1877). S. 1-2, hier S. 2. 
„der katholische Charakter der österreichischen Universitäten definitiv aufgehoben“" worden sei. ${ }^{49}$

Umgekehrt lobten beispielsweise die beiden katholisch-konservativen Blätter, die Neuen Tiroler Stimmen ${ }^{50}$ oder das Linzer Tagblatt, das Engagement und den Durchhaltewillen der Innsbrucker Studenten, vor allem die Umsicht der Theologen, und verwiesen darauf, dass bei zahlreichen Festen anderer Universitäten (München, Bonn, Agram) Gottesdienste zum offiziellen Programm gehört hätten, was überdies der Tradition der Universität entspräche. Gleichzeitig verurteilte man die liberale Presse und deren ,jüdische Preßharpyen“, und unterstellte ihr, dass sie mit „Fanatismus“ von Wien aus danach trachte, der Innsbrucker „Alma mater gelegentlich ihres Jubiläums den rothen Hahn des Parteizwistes auf's Dach zu setzen“. ${ }^{51} \mathrm{Zu}$ dem Vorwurf, die Tradition der Universität zu missachten, kommt damit auch das Motiv einer Einmischung von außen.

Da die Universität dadurch eine öffentliche Aufmerksamkeit erhalten hatte, die kein gutes Licht auf sie warf, zog Rektor Huber die Notbremse und versagte die Benützung der Aula am Festtag, und begründete dies damit, dass „ein großer

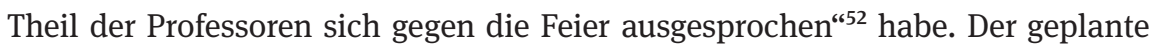
Fackelumzug musste ebenfalls abgesagt werden, weil ihn der (konservative) Statthalter Eduard Taaffe nicht bewilligte mit dem Argument, die Studenten hätten den Fackelzug zu spät angemeldet. In der liberalen Presse wurde allerdings vermutet, dass der Statthalter die Feier der Theologen „patronisiert“ habe. ${ }^{53}$

In Reaktion auf diese Widrigkeiten fasste das Festkomitee der Studierenden den Beschluss „von einer gemeinsamen Feier des 200-jährigen Bestandes unserer Universität abzusehen. “54 So wurden am 26. April, einem Donnerstag und dem Tag der Ausstellung der kaiserlichen Bestätigung der Universität, kleinere, getrennte Feiern abgehalten, wobei die Gruppe der Theologiestudenten und der katholischen Verbindungen Austria und Helvetia den so umkämpften Gottesdienst in der Universitätskirche veranstalteten, zu dem man in einem Umzug mit 15 Wagen vorfuhr. Nach der Festmesse gab es ein gemeinsames Mahl. Auch Besucher von außerhalb fanden sich ein, namentlich genannt wird in den Berichten die katholische Verbindung Aenania aus München. Am Nachmittag besuchte

49 O.V., Innsbruck (wie Anm. 48), S. 2.

50 O.V.: Die Universitätsfeier. In: Neue Tiroler Stimmen Nr. 93 (24.4.1877). S. 3.

51 O.V., Akademisches (wie Anm. 44), S. 3.

52 UAI, Senatssitzungsprotokolle 1862-1885, Karton 4, V 1876/77: Senatssitzung vom 5.5.1877.

53 O.V., Die Schwarzen (wie Anm. 43); ähnlich auch: o.V.: Innsbruck. In: Prager Tagblatt Nr. 113 (24.4.1877). S. 4 .

54 O.V.: Die partielle Universitätsfeier. In: Innsbrucker Nachrichten Nr. 96 (27.4.1877). S. 1180 1181, hier S. 1180. 
die Gruppe den Bergisel, „um den Tag durch erhebende Erinnerung an die ruhmvollen Thaten der Vergangenheit zu feiern“.55 Am Bergisel hatten im Jahr 1809 die Tiroler Aufständischen gegen bayerische und französische Heere gekämpft und dieser Ort ist bis heute ein Tiroler Gedächtnisort. Indem der damalige Aufstand stets auch als Kampf des katholischen Tiroler Volkes gegen die kirchenund religionsfeindliche Politik des mit dem napoleonischen Frankreich verbündeten bayerischen Königs gedeutet worden war, konnte dies in die evozierte katholische und gegenreformatorische Tradition der Universität eingepasst werden. Am Abend des 26. April folgten getrennte Festkommerse der beiden Gruppen, die schließlich in den eingangs erwähnten Schlägereien in der Innenstadt endeten. ${ }^{56}$

Der Universitätssenat arbeitete diese Vorfälle wenige Tage später auf, wobei es vor allem darum ging, inwiefern der Rektor energischer gegen die Vorhaben der Studenten hätte einschreiten müssen. Huber rechtfertigte sich damit, dass er sich stets im Rahmen der gesetzlichen Vorgaben bewegt sowie mehrfach versucht habe, die Studenten zum Verzicht auf das Fest zu bewegen und durch das Verbot, die Aula zu benützen, einen größeren Skandal verhindert habe. Allerdings musste Huber zugeben, dass das Ereignis unnötiges Aufsehen erregt und den Ruf der Universität, der durch den Streit der Professoren ohnehin gelitten hätte, beschädigt habe. ${ }^{57}$

Die Studenten besprachen wenige Tage später die Situation ebenfalls, wobei sie sich bei dieser Sitzung durch die Anwesenheit des Rektors brüskiert fühlten und daher mit großer Mehrheit beschlossen, sich an keiner weiteren Jubiläumsfeier, sollte eine solche vom Senat geplant werden, zu beteiligen. ${ }^{58}$ Ein solcher Plan für eine Feier im November 1877 war tatsächlich kurz im Senat diskutiert, aber, wohl auch unter dem negativen Eindruck der Ereignisse, wieder fallen gelassen worden. ${ }^{59}$

Auch in der Presse gab es in der Folge noch einige Kommentare zu der Feier, wobei sich ein Redakteur im St. Pöltner Boten, einem Organ des katholisch-pa-

55 O.V., Universitätsfeier (wie Anm. 54), S. 1180.

56 Eine ausführliche Beschreibung des Tages findet sich in: o.V., Universitätsfeier (wie Anm. 54); es folgten in den nächsten Tagen noch mehrere Berichte über die nächtlichen Auseinandersetzungen: o.V.: Von der Universitätsfeier. In: Innsbrucker Nachrichten Nr. 97 (28.4.1877). S. 1189; o.V.: Zum Studentenkrawall. In: Innsbrucker Nachrichten Nr. 98 (30.4.1877). S. 1207.

57 UAI, Senatssitzungsprotokolle 1862-1885, Karton 4, V 1876/77: Senatssitzung vom 5.5.1877. 58 UAI, Senatssitzungsprotokolle 1862-1885, Karton 4, ad V 1876/77: Rektor Alfons Huber an Ministerium für Cultus und Unterricht. 9.5.1877.

59 UAI, Senatssitzungsprotokolle 1862-1885, Karton 4, V 1876/77: Senatssitzung vom 5.5.1877; Anlass dazu hätte die öffentliche Verkündung der Stiftungsurkunden in der Jesuitenkirche am 16. November 1677 gegeben. 
triotischen Volks- und Pressvereins in Niederösterreich, ${ }^{60}$ besonders hervortat. Er erörterte zunächst die bekannte Sachlage und erklärte, dass sich alle Professoren und ein Großteil der Studenten aufgrund der Aufnahme eines Gottesdienstes in das Festprogramm von der Feier distanziert hätten, um dann festzustellen, dass die Feier dennoch ,glänzend ausfiel“, weil die katholischen Studenten zusammengehalten hätten. Auch in diesem Artikel findet sich eine Verbindung von Vergangenheit und Gegenwart, indem der Streit als Symptom der Gegenwart gedeutet wird:

\begin{abstract}
Allein wir fragen, was soll aus dem kath. Oesterreich werden, wenn die zukünftigen Beamten, Professoren und Doktoren eine solche Abneigung gegen die Religion haben, daß sie nicht einmal ein Hochamt vertragen!? Das Ganze beweist wieder, daß wir Recht haben: Die Entchristlichung der Jugend nimmt zu, selbst in Tirol. Wie es wo anders ist, wissen wir leider zu gut! ${ }^{61}$
\end{abstract}

Weil anders als bei späteren Jubiläen aufgrund der geschilderten Konflikte keine offizielle Festschrift entstand, blieb von diesem kontroversen 1877er-Jubiläum wenig Dauerhaftes. Für eine Festschrift fehlten sowohl die Vorbereitungszeit als auch das gemeinschaftliche Interesse der Professoren sowie ein gemeinsames Narrativ, auf dem eine solche Festschrift hätte aufbauen können. Indes haben die Studenten auch diese Leerstelle erkannt und versucht, einen gewissen Ersatz zu schaffen, sodass, nebst der Presse mit ihren Artikeln, die Studenten auch überwiegend für die wenigen schriftlichen Produkte im Umfeld des Jubiläums verantwortlich zeichneten. Der Germanistenklub und das sogenannte „WaltherDenkmal-Comité“, das indes nicht aus Studenten bestand, veröffentlichten eine Studie mit dem Titel „Gedichte Rubins“62. Bis auf einen kurzen Verweis auf die „hohe Schule, [die] der Innstrom wild umrauscht“ in einem Widmungsgedicht des fromm-sentimentalen Dichters und zeitweiligen Professors für Literatur und Ästhetik in Wien Oskar Redwitz, handelt das Buch aber ausschließlich vom Minnesänger Rubin und dessen herausgestellter Tiroler Herkunft. Außerdem widmete der Student Joseph Wackernell seine Studie zu Walther von der Vogelweide, die zugleich seine Dissertation war und die er zu diesem Zeitpunkt ohnehin fertiggestellt hatte, der Universität „zur 200jährigen Stiftungsfeier“. ${ }^{63}$ Während diese

60 Günter, Johann: Das niederösterreichische Pressewesen von 1848-1918 mit Ausnahme Wiens. Dissertation. Wien 1973. S. 36.

61 O.V.: Innsbruck. In: St. Pöltner Bote Nr. 18 (3.5.1877). S. 146.

62 Gedichte Rubins. Bozen 1877 (Publicationen des Walther-Denkmal-Comité in Bozen. Zum Besten des Denkmal-Fondes). Die Herausgeberschaft durch den Germanistenklub der Universität und das Walther-Denkmal-Comité wird erklärt in: o.V., Universitätsfeier (wie Anm. 50).

63 Wackernell, Joseph E.: Walther von der Vogelweide in Oesterreich. Innsbruck 1877. 
ersten beiden Veröffentlichungen somit den Charakter von kurzfristigen Verlegenheitsgaben besitzen, gab es indes auch eine Publikation, die besonders hervorzuheben ist, weil sie in direktem Bezug zur Universität und in das Genre der durch Universitätsjubiläen angeregte Geschichtsschreibung passt: ein Büchlein, das der Geschichtsstudent Josef Innerhofer seinen „Collegen“ gewidmet hatte, ${ }^{64}$ versammelte auf 32 Seiten die Texte der Stiftungsurkunden von Kaiser Leopold und Papst Innozenz XI. in Übersetzung sowie „einige Skizzen aus der Geschichte der Universität““ ${ }^{65}$ Dort wird vorrangig die Gründungsgeschichte erläutert und dabei vor allem auf die ersten Statuten eingegangen, die „einen wesentlich katholischen Charakter trugen“. ${ }^{66}$ Die Epoche der Aufklärung, in der sich „der ,freie Geist' einen zweifelhaften Ruhm an der tirolischen Hochschule bereitete" ${ }^{67}$ kam hingegen schlecht weg und somit lässt sich erahnen, welchem Teil seiner Kollegen der Autor diesen Band wohl in erster Linie widmete.

\section{Fazit}

Die Jahre vor 1877 waren in Innsbruck geprägt von Konflikten hinsichtlich der öffentlichen Positionierung der Universität und ihrer Identität. Einzelne oder mehrere Fakultäten und Professoren hatten versucht, die Position und Funktion der Universität bei öffentlichen Anlässen mehrfach und unterschiedlich zu definieren. Der zentrale Streitpunkt war dabei die Rolle der Kirche in der Universität. Ein Jubiläum, ein Ereignis mit besonderem Prestige, wäre in diesem Sinne eine einzigartige Möglichkeit gewesen, mit einem Bekenntnis zu einer gemeinsamen Tradition eine Positionierung für die Gegenwart vorzunehmen, was aus geschilderten Gründen aber nicht erreicht werden konnte.

Die Gründe für das Scheitern lagen vor allem in internen Streitigkeiten der Universität um das Finden eines gemeinsamen Programms und Narrativs für die Feier. Gleichzeitig kann man diesen Konflikt in einem größeren Rahmen der Auseinandersetzung um die Rechte der Kirche innerhalb der Habsburgermonar-

64 Innerhofer, Josef: Gedenkblätter an die zweihundertjährige Jubelfeier der k. k. Universität Innsbruck. Seinen Collegen gewidmet. Innsbruck 1877; die Arbeit beruht in erster Linie auf der Geschichte der Universität von Jacob Probst, die 1869 - auch in Reaktion auf die „tabula rasa“, die damals im österreichischen Bildungssystem gemacht worden war - erschienen ist. Probst, Jacob: Geschichte der Universität Innsbruck seit ihrer Entstehung bis zum Jahre 1860. Innsbruck 1869; das Zitat ebd. S. 341.

65 Innerhofer, Gedenkblätter (wie Anm. 64), S. 21.

66 Innerhofer, Gedenkblätter (wie Anm. 64), S. 25.

67 Innerhofer, Gedenkblätter (wie Anm. 64), S. 28. 


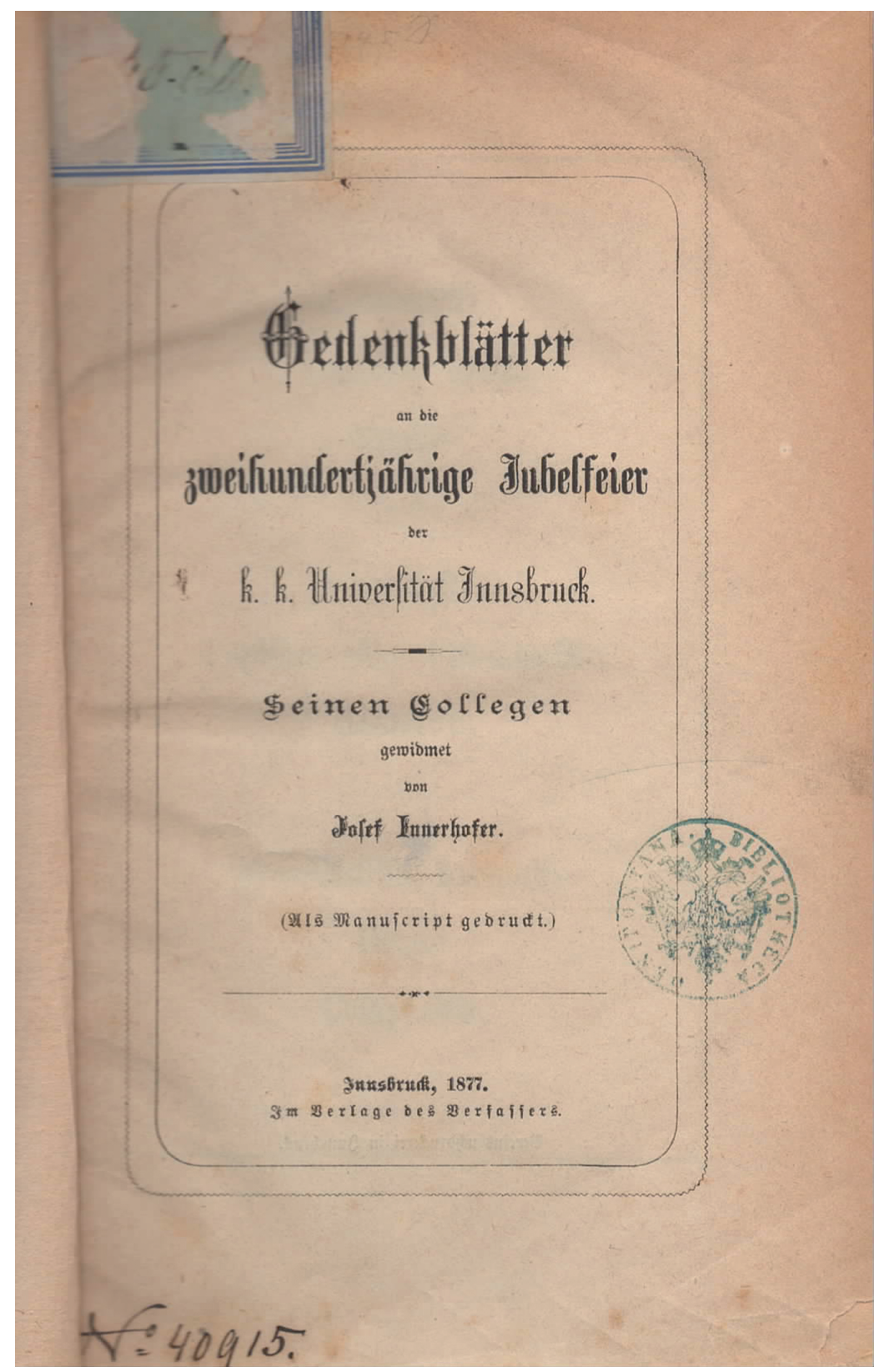

Abb. 3: Das Bild zeigt die Titelseite der einzigen Publikation zum Universitätsjubiläum, die die Universität und deren Geschichte selbst behandelt. Sie wurde vom Studenten Josef Innerhofer verfasst. Universitätsbibliothek Innsbruck. 
chie in den Jahrzehnten nach 1848 verorten. Das Bündnis von Thron und Altar in der Folge der Revolution, gipfelnd im Konkordat von 1855, hatte der Kirche wieder eine dominante Stellung in Staat und Gesellschaft sowie zahlreiche Vorrechte im Bildungswesen eingeräumt. In der liberalen Ära nach 1860 war diese Vormachtstellung der Kirche von Liberalen und Deutschnationalen indes in Frage gestellt und bekämpft worden, was schließlich in die Aufkündigung des Konkordats nach der Verkündung des Unfehlbarkeitsdogmas durch den Papst mündete. Gerade in Tirol hatte diese neuerliche Entmachtung der Kirche sowie die Gewährung der Glaubensfreiheit in der Verfassung von $1867 \mathrm{zu}$ heftigen Protesten geführt, die letztlich auch in der Spaltung der Universität sowie in den Vorbereitungen zum Jubiläum sichtbar werden.

Das gescheiterte Jubiläum der Universität Innsbruck beziehungsweise die chaotischen Planungen desselben sind somit wie ein Brennglas, in dem sich die Konflikte und Debatten innerhalb der Monarchie sowie an der Universität Innsbruck in diesen Jahren fokussieren und deutlich hervortreten. Überdies zeigt das Gedenkjahr Praktiken der Erinnerung, unterschiedliche Formen der Inszenierung des Jubiläums durch die Studenten und die jeweils aufgegriffenen Traditionen. Geeint wurden die Versuche letztlich durch das Scheitern ihres Anspruchs, universitäre Gemeinschaft zu repräsentieren und eine einende Identität zu stiften. Auch die wohl prestigeträchtigste, weil dauerhafteste, Form ein Jubiläum zu feiern, die Herausgabe einer Festschrift, kam nicht zustande. Damit fiel zudem ein bei anderen Jubiläen wichtiger Effekt weg, nämlich die Produktion von neuem Wissen über die Universität durch ein Jubiläum. Hier wird besonders augenfällig, dass die im Normalfall dominantesten Akteure innerhalb der Universität, die Professoren, beim Jubiläum keine Rolle spielten und vielmehr als Verhinderer der Feiern galten.

Der zentrale Ort der Planung der Feier war die Studentenversammlung. Darüber hinaus trugen Studenten die Debatten von dort in die Öffentlichkeit, indem sie die Presse immer wieder über die Vorkommnisse an der Universität informierten. Das selbstbewusste Auftreten der Studentenschaft gegenüber dem Rektorat und die Tatsache, dass den Studenten ihre Handlungsmacht bewusst wurde, ist letztlich auch eine zentrale Folge des gescheiterten Jubiläums. In den kommenden Jahren und Jahrzehnten avancierten die Studenten zu einem wichtigen Faktor in der Universitätspolitik.

Für die Professoren bot sich erst zwei Jahrzehnte später die Chance, das misslungene Jubiläum von 1877 wettzumachen. Die Gelegenheit dazu bot das 50jährige Thronjubiläum von Kaiser Franz Joseph im Jahr 1898. Die Integrationsfigur des Kaisers musste damit als Ersatz für das verpasste Jubiläum zwei Jahrzehnte zuvor dienen. Anlässlich dieses Ereignisses legte die Professorenschaft eine 
prachtvolle Festschrift zu der Geschichte der Universität vor. ${ }^{68}$ In dieser zeigt sich denn auch ein weitgehend gefestigtes Bild der Innsbrucker Universität als Staatsanstalt, die für gesellschaftlichen und wissenschaftlichen Fortschritt stand und die sich als Institution einer, deutschen Wissenschaft' in einem gemischtsprachigen Kronland verstand. Diese Position schloss damit ebenfalls eine Gruppe aus, nämlich die italienischsprachige Minderheit des Landes. Mit dieser Abwehrhaltung gegenüber einer beargwöhnten italienischen „Überfremdung“ und der Ausgrenzung einer Minderheit konnte nunmehr die 1877 vermisste Gemeinschaft hergestellt und die zuvor unüberwindbar scheinenden Gräben zugeschüttet werden. ${ }^{69}$

68 Akademischer Senat (Hrsg.): Die Leopold-Franzens-Universität zu Innsbruck in den Jahren 1848-1898. Festschrift aus Anlass des 50-jährigen Regierungsjubiläums Sr. Majestät des Kaisers Franz Joseph I. Innsbruck 1899.

69 Für folgende, ebenfalls nicht friktionsfreie Jubiläen der Universität siehe Goller, Universitätsjubiläen (wie Anm. 33). 
